\title{
Switched Control of a Time Delayed Compass Gait Robot
}

\author{
Elyes Maherzi \\ National School of Engineers of \\ Carthage, \\ University of Carthage, \\ Research Unit: Signals and \\ Mechatronic Systems \\ Tunis, Tunisia
}

\author{
Walid Arouri \\ Higher Institute of Industrial \\ Systems \\ University of Gabes
}

\author{
Mongi Besbes \\ Higher Institute of Information and \\ Communication Technologies \\ University of Carthage, \\ Research Unit: Signals and \\ Mechatronic Systems \\ Tunis, Tunisia
}

\begin{abstract}
- the analysis and control of delayed systems are becoming more and more research topics in progress. This is mainly due to the fact that the delay is frequently encountered in technological systems. Most control command laws are based on current digital computers and delays are intrinsic to the process or in the control loop caused by the transmission time control sequences, or computing time. In other hand, the controls of humanoid walking robot present a common problem in robotics because it involves physical interaction between an articulated system and its environment. This close relationship is actually a common set of fundamental problems such as the implementation of robust stable dynamic control. This paper presents acomplete approach, based on switched system theory, for the stabilization of a compass gait robot subject to time delays transmission. The multiple feedback gains designed are based on multiple linear systems governed by a switching control law. The establishment of control law in real time is affected by the unknown pounded random delay. The results obtained from this method show that the control law stabilize the compass robot walk despite a varying delay reaching six times sampling period.
\end{abstract}

Keywords-Biped robot; delayed system; Switched system; Stability; Lagrange formulation; Lyapunov method; Relaxation; Linear matrix inequalities (LMI); bilinear matrix inequalities (BMI)

\section{INTRODUCTION}

Research on mobile robots during the last three decades has a huge progress. The biped robots are a relevant class of mobile robots due to their adaptability to various floors grounds and movement in rough environments. The nonlinearity of biped walking makes the conventional control methods obsolete.

The stable walking of a biped robot can be defined as a stable oscillation around dynamic equilibrium points [1]. Other researchers are based mainly on the decomposition of a gait cycle indifferent main phases; flight, single and double support, with instant impact phase [2]. In this case the objective is to find a stabilizing control law which run between multiple operating modes, where each mode is governed by its own dynamics. The overall feedback control must stabilize each mode separately and the transitions between them. Therefore, we can formulate a switched model that includes the description of different mode and switching between them [3].
The study of the stability of biped robots under the effect of communication delays is currently the subject of intense research in the branch of the automatic delayed systems. By the way, research in the field of systems controlled via computer networks is growing. The analysis and control synthesis of delay systems are becoming more and more research topics in progress [4] [5].This is mainly due to the fact that the delay is frequently encountered in technological systems and can affect their behaviors significantly. Most are base don current digital computers and delays may occur intrinsically to the process or in the control loop caused by the transmission time control sequences, or computing time. The delay may affect one or more states of the considered system. It may also affect the establishment of the output. Several studies have modeled the linear systems with delays by differential equations covering both the present and the past states of the system, assuming that the derivative of the vector of states can be explained at every time t. Other studies consider delay systems as nonlinear and no stationary [6], [7] with parameters varying depending on time or the state of the system. The representation of such variation may be continuous or piecewise continuous [8].Modeling a delayed discrete time system as switched system is a new approach emerging from researches on lines supports and telecommunications systems. The idea is to build a set of several systems where each set constraints a value of delay[9]. Applied to the case of biped walking the overall model must be represented by a switched system submitted to two switching law. The first one is depending on gait cycle phases and known in real time, which allow us to choose between the appropriate feedback gains. The second one is unknown and depending on the delay value, which is bounded and integer (multiple of the sampling time). The feedback control synthesis approach is considered as a problem of robust control and leads us to aset of non-linear matrix inequalities conditions. To overcome this difficulty, we propose original relaxations stabilizing the robot running despite the delays and the non-linearity's.

In the first part of this article, we present a feedback control synthesis method for the command of delayed discrete time systems, based on the second method of Lyapunov.

The second part is dedicated to the modeling of compass gait biped robot. In the last part we show the results of the method applied to the obtained model. 


\section{STABILIZABILITY OF A DELAYED SYSTEM}

\section{A. Stability and stabilization of switched system}

The stability of switched system analysis is assumed using a sufficient (but relatively nonrestrictive compared to the quadratic approach) stability condition using the poly-quadratic approach [10][11]. This approach is drawn primarily from a parameter dependent Lyapunov function [12].

Let's consider the following switched system defined as hybrid systems represented by a set whose elements are dynamic discrete time models with commutation law which define, in time, the switch between the elements:

$$
x(k+1)=\sum_{i=1}^{N} \mu_{i}(k) A_{i}(k) x(k)+\sum_{i=1}^{N} \mu_{i}(k) B_{i}(k) u(k)
$$

Where the parameters $\mu_{i}(k)$ replace the commutative law such as $\sum_{i=1}^{N} \mu_{i}=1$ the feedback control is written in the following form:

$$
u(k)=\sum_{i=1}^{N} \mu_{i}(k) K_{i}(k) x(k)
$$

The closed loop system is described by the following equation:

$$
x(k+1)=\sum_{i=1}^{N} \mu_{i}(k)\left(A_{i}+B_{i} K_{i}\right) x(k)
$$

The poly-quadratic stability analysis of the switched systems was proposed by [10]. It is possible to write the system (3) under the same following expression

$\xi_{i}=\left\{\begin{array}{l}1: \text { If the model is described by the matrix } \mathrm{A}_{i} \\ 0: \text { otherwise }\end{array}\right.$

$$
A\left(\xi_{k}\right)=\sum_{i=1}^{N} \xi_{k}^{i} A_{i} ; \xi_{k}{ }^{i} \geq 0 ; \sum_{i=1}^{N} \xi_{k}{ }^{i}=1
$$
form:

We can thus write the system according to the following

$$
x(k+1)=\sum_{i=1}^{N} \xi_{k}{ }^{i} A_{i}
$$

The system (5) is poly-quadratically stable only if there are $\mathrm{N}$ symmetric matrices defined positively $S_{l} \ldots S_{N}$ and $\mathrm{N}$ matrices $G_{1} \ldots G_{N}$ of appropriate dimensions confirming:

$$
\left[\begin{array}{cc}
G_{i}+G_{i}^{T}-S_{i} & G_{i}^{T} A_{i}^{T} \\
A_{i} G_{i} & S_{j}
\end{array}\right]>0, \forall i, j \in\{1 \ldots N\}
$$

The parameter dependent Lyapunov function used is written as:

$$
\mathrm{V}(x(\mathrm{k}) \xi(k))=x^{t}(k) \sum_{i=1}^{N} P_{i}\left(\xi_{k}^{i}\right) x(k)
$$

With: $P_{i}=S_{i}^{-1}$

Replacing $A_{i}$ by $\left(A_{i}+B_{i} K_{i}\right)$ and linearizing the matrix disparity by the change of variable $R_{i}=G_{i} K_{i}$. We reach the following condition expressed in LMI terms:

$$
\left(\begin{array}{cc}
G_{i}+G_{i}^{t}-S_{i} & G_{i}^{t} A_{i}^{t}+R_{i}^{t} B_{i}^{t} \\
A_{i} G_{i}+B_{i} R_{i} & S_{j}
\end{array}\right)>0
$$

$\forall i, j \in\{1 \ldots N\}$

The closed loop system is asymptotically stabilizable by state feedback if there are symmetric matrices $S_{i j} \succ 0$, Matrices $R_{i}, G_{i}$ of appropriate dimensions such as the gain of return of state is given by:

$$
K_{i}=R_{i} G_{i}^{-1}
$$

\section{B. Stability analysis of delayed switched system}

When the delay affects commands, $u(k)=K_{i} x(k-\tau(k))$ where $\tau(k) \in\left[\tau_{\min }, \tau_{\max }\right]=\left[i_{\min } T_{e}, i_{\max } T_{e}\right]$ is a variable delay.

Then we consider the augmented state vector:

$$
\chi(k)=\left[x(k)^{T} \ldots x\left(k-\tau_{\min }\right)^{T} \ldots x\left(k-\tau_{\max }\right)^{T}\right]^{T}
$$

Condition (6) can be used for the stability analysis of discrete delay system. The equivalence between the LyapunovKrasovskii functional approach for discrete delay systems and the stability conditions (6) was proved in [13].

The dynamic of the system can be represented by set of a state matrix:

$$
\bar{A}_{i}=\left[\begin{array}{cccccccc}
A_{i} & 0 & \ldots & 0 & B_{i} K_{i} & 0 & \cdots & 0 \\
I & 0 & \ldots & \ldots & \ldots & \cdots & \cdots & 0 \\
0 & I & 0 & \cdots & \cdots & \cdots & \cdots & 0 \\
\vdots & & & & & & & \vdots \\
0 & \ldots & \ldots & \ldots & \ldots & 0 & I & 0
\end{array}\right]
$$

The position of $B_{i} K_{i}$ is depending in the value of $i$, it is found on the $(i+1)^{t h}$ column of the first line of $\bar{A}_{i}$.

The augmented switched discrete delay system can be seen as an uncertainty switched system. Where any element of the set is a polytope whose apex are depending on the value of delay as shown in figure 1 . Where $S S_{n}$ represent the subsystems of the delayed switched system. $A_{n i}$ the different apex $\bar{A}_{i}$ of the subsystem n.

Then the condition of stability analysis of the switched discrete delay system is the same than the one used for a polytopic uncertainty switched system [14]. 


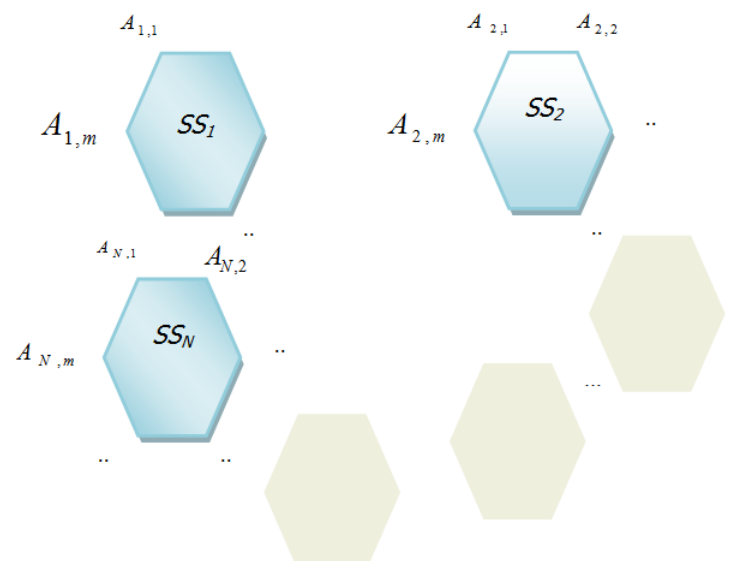

Fig. 1. Uncertainty switched system

Considering in condition (6) a commune matrix $G$ of appropriate dimensions:

$$
G=\left[\begin{array}{ccccc}
g_{1,1} & g_{2,1} & . & . . & g_{1, i_{\max }+1} \\
. . & . & . & . & . \\
\cdot & . . & . & . . & . \\
g_{i_{\max }+1,1} & . & . . & . & g_{i_{\max }+1, i_{\max }+1}
\end{array}\right]
$$

The condition (6) becomes:

$$
\begin{gathered}
{\left[\begin{array}{cc}
G+G^{t}-S_{i} & \Xi_{i}^{T} \\
\Xi_{i} & S_{j}
\end{array}\right]>0} \\
\forall i, j \in\left[\tau_{\min } / T_{e}, \tau_{\max } / T_{e}\right]
\end{gathered}
$$

With:

$$
\Xi_{i}=\left[\begin{array}{ccccc}
A_{i} g_{1,1}+B_{i} K_{i} g_{i+1,1} & . & . & . & A_{i} g_{i_{\max }+1}+B_{i} K_{i} g_{i+1, i_{\max }+1} \\
g_{1,1} & g_{1,2} & . & . & g_{1, i_{\max }+1} \\
. & . & . & . & . \\
. & . . & . & . & . \\
g_{i_{\max }, 1} & . & . & . & g_{i_{\max }, i_{\max }+1}
\end{array}\right]
$$

The condition (13) is a $B M I$, which is impossible to solve by the current solvers. That is why it is essential to introduce relaxations in order to obtain linear conditions:

First we start with the change of variables $R_{i}=K_{i} g_{i+1, i+1}$, then we consider $g_{i, j}=0 \forall i \neq j$ for all $i, j \in\left[\tau_{\min } / T_{e}, \tau_{\max } / T_{e}\right]$.

Then we replace in the conditions (13) $\Xi_{i}(14)$ by:

$$
\Xi_{i}=\left[\begin{array}{ccccc}
A_{i} g_{1,1} & 0 & A_{i} g_{i+1, i+1}+B_{i} R_{i} & . & 0 \\
g_{1,1} & 0 & . & . & 0 \\
\cdot & . & . \cdot & . & \cdot \\
\cdot & . \cdot & . & . & \cdot \\
0 & . & . & g_{i_{\max }, i_{\max }} & 0
\end{array}\right]
$$

The feedback gains are given by: $K_{i}=R_{i} g_{i+1, i+1}^{-1}$

In case we are looking for a common feedback gain:

$$
K=R_{i} g_{i+1, i+1}^{-1} \forall i \in\left[\tau_{\min } / T_{e}, \tau_{\max } / T_{e}\right]
$$

Then it is necessary to satisfy the equality (16), to overcome this non-linearity one can consider:

$$
R_{i}=R_{j}, g_{i, i}=g_{j, j} \forall i, j \in\left[\tau_{\min } / T_{e}, \tau_{\max } / T_{e}\right]
$$

The matrix $G$ becomes:

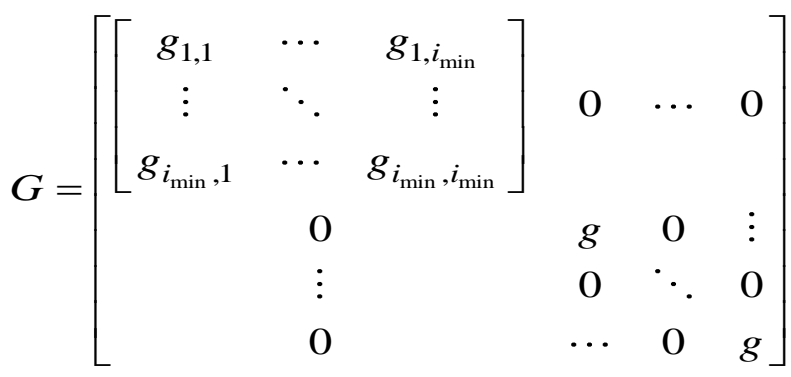

In particular case where $\tau_{\text {min }}=0: G=g \times I_{i_{\max } \times i_{\max }}$

with $I$ is the identity matrix of appropriate dimensions.

\section{SWITCHED SYSTEM MODEL FORWALKING COMPASS GAIT ROBOT}

In this part, we are interested, in the modeling of the walking compass gait biped robot. The control sequence is transferred via a network data transmission. This transfer prevents the establishment of command sequence in the real time. We study more particularly the linear system stability when the pair applied by actuators, switches between the hip, the ankle, or the pair ankle - hip at the same time.

First, we have considered as a walker simplified model a bi-pendulum formed of point masses, containing only a single articulation in the hip, which is capable of reproducing the running. It is called compass gait robot. The figure 1 presents this geometrical conception of the compass robot (fig.2). [15]

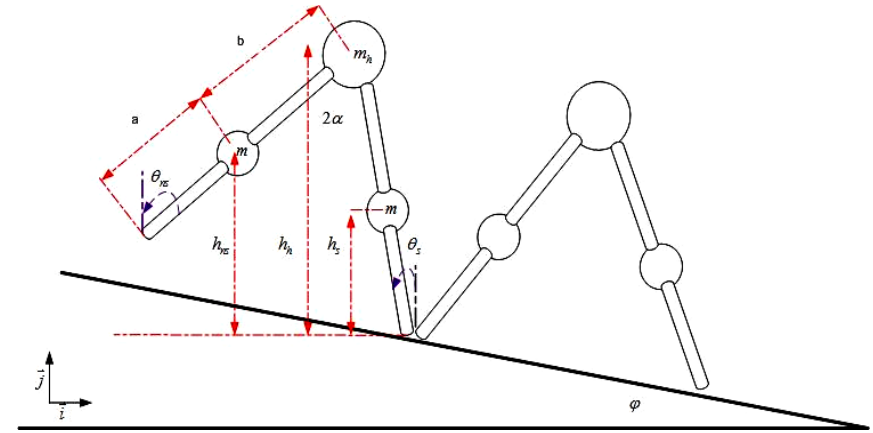

Fig. 2. Compass robot

$\theta_{s}:$ Absolute angle of the leg in touch with the ground (indication ' $\mathrm{s}$ ' is for support leg) 
$\theta_{n s}:$ Absolute angle of the leg during flight (indication 'ns' is for no swing leg)

$\alpha$ : The half inter leg angle

$\varphi$ : The slope angle

$h_{n s}, h_{s}$ : Height separating both legs with regard to the point of biped contacting the ground

$\mathrm{h}_{h}$ : Height between hip and the point contacting the sole of compass

$\mathrm{m}, \mathrm{m}_{h}$ : Masse of the pendulums which represents the leg and the hip

The equations of the compass during the phase of simple support are obtained by using the following Equations of Euler-Lagrange:

$$
\frac{d}{d t}\left(\frac{\partial L(\dot{\theta}, \theta)}{\partial \dot{\theta}}\right)-\left(\frac{\partial L(\dot{\theta}, \theta)}{\partial \theta}\right)=F
$$

With: $L(\dot{\theta}, \theta)$ : the Lagrangian of the system :

$$
L(\dot{\theta}, \theta)=E_{c}(\dot{\theta}, \theta)-E_{p}(\dot{\theta}, \theta)
$$

$F$ : External forces applied to the system.

The correspondent relations between the actuator pairs and the robot degrees of freedom are represented as follows:

$$
\frac{\partial P_{u}}{\partial \dot{\theta}}=\operatorname{Torc}_{i}
$$

The Power $P_{u}$ is given by the following relation:

$$
\begin{aligned}
& P_{u}=T_{c} \dot{\theta}_{s}+T_{h}\left(\dot{\theta}_{s}-\dot{\theta}_{n s}\right) \\
& \frac{\partial P_{u}}{\partial \dot{\theta}_{s}}=T_{c}+T_{h} \\
& \frac{\partial P_{u}}{\partial \dot{\theta}_{n s}}=T_{c}-T_{h}
\end{aligned}
$$

The equations of Euler-Lagrange are written by:

$$
M(\theta) \ddot{\theta}+N(\theta, \dot{\theta})+G(\theta)=J * \operatorname{Torc}_{i}
$$

With:

$$
\begin{aligned}
& M(\theta)=\left(\begin{array}{cc}
m b^{2} & -m b l \cos \left(\theta_{s}-\theta_{n s}\right) \\
-m b l \cos \left(\theta_{s}-\theta_{n s}\right) & m_{h} l^{2}+m\left(a^{2}+l^{2}\right)
\end{array}\right) \\
& N(\theta)=\left(\begin{array}{l}
-m b l \sin \left(\theta_{s}-\theta_{n s}\right) \dot{\theta}_{n s}^{2} \\
m b l \sin \left(\theta_{s}-\theta_{n s}\right) \dot{\theta}_{s}^{2}
\end{array}\right) \\
& G(\theta)=\left(\begin{array}{l}
m g b \sin \left(\theta_{n s}\right) \\
-\left(m_{h} l+m g(a+l) \sin \left(\theta_{n s}\right)\right)
\end{array}\right) ; J=\left(\begin{array}{cc}
-1 & 0 \\
1 & 1
\end{array}\right) \\
& \operatorname{Torc}_{i}=\left(\begin{array}{l}
T_{h} \\
T_{c}
\end{array}\right)
\end{aligned}
$$

The torc is applied to the hip and the ankle.

The Lagrange equation (1) can, thus, be written in the following form:

$$
\begin{aligned}
& \left(\begin{array}{cc}
m b^{2} & -m b l \cos \left(\theta_{s}-\theta_{n s}\right) \\
-m b l \cos \left(\theta_{s}-\theta_{n s}\right) & m_{h} l^{2}+m\left(a^{2}+l^{2}\right)
\end{array}\right)\left(\begin{array}{l}
\ddot{\theta}_{n s} \\
\ddot{\theta}_{s}
\end{array}\right)+ \\
& \left(\begin{array}{l}
-m b l \sin \left(\theta_{s}-\theta_{n s}\right) \dot{\theta}_{n s}{ }^{2} \\
m b l \sin \left(\theta_{s}-\theta_{n s}\right) \dot{\theta}_{s}^{2}
\end{array}\right)+\left(\begin{array}{l}
m g b \sin \left(\theta_{n s}\right) \\
-\left(m_{h} l+m g(a+l) \sin \left(\theta_{n s}\right)\right)
\end{array}\right)=J\left(\begin{array}{l}
T_{h} \\
T_{c}
\end{array}\right)
\end{aligned}
$$

The state vector $q=\left(\begin{array}{c}\theta_{n s} \\ \theta_{s} \\ \dot{\theta}_{n s} \\ \dot{\theta}_{s}\end{array}\right)$ the linear representation of the compass model by the jacobian method is thus written as:

$$
\dot{q}=\left(\begin{array}{cccc}
0 & 0 & 1 & 0 \\
0 & 0 & 0 & 1 \\
\ldots & A_{j a c o b} & \cdots
\end{array}\right)\left(\begin{array}{l}
\theta_{n s} \\
\theta_{s} \\
\dot{\theta}_{n s} \\
\dot{\theta}_{s}
\end{array}\right)
$$

With:

$$
A_{j a c o b}=\frac{\partial}{\partial q}\left[\left(-M(\theta)^{-1} N(\theta, \dot{\theta})-M(\theta)^{-1} G(\theta)\right)\right]_{q=q_{e}=0}
$$

The linear representation of the compass model is written as follows:

$$
\begin{aligned}
& \dot{q}=\left(\begin{array}{l}
\dot{\theta}_{n s} \\
\dot{\theta}_{s} \\
\ddot{\theta}_{n s} \\
\ddot{\theta}_{s}
\end{array}\right)=\left(\begin{array}{cccc}
0 & 0 & 1 & 0 \\
0 & 0 & 0 & 1 \\
\left(m_{h} l^{2}+m\left(a^{2}+l^{2}\right)\right) m g & -m b l\left(m_{h} l+m(a+l) g\right) & 0 & 0 \\
m^{2} b^{2} l \mathrm{~g} & -m b l\left(m_{h} l+m(a+l) g\right) & 0 & 0
\end{array}\right)\left(\begin{array}{l}
\theta_{n s} \\
\theta_{s} \\
\dot{\theta}_{n s} \\
\dot{\theta}_{s}
\end{array}\right) \\
& +\left(\begin{array}{ll}
0 & 0 \\
0 & 0 \\
. . J . .
\end{array}\right)\left(\begin{array}{l}
T_{h} \\
T_{c}
\end{array}\right)
\end{aligned}
$$

The pair applied by actuators, switches between the hip, ankle, or the pair ankle - hip at the same time. This switching is described by the selection matrix $J$.

- Hip is commanded: In this case, where only the hip is commanded, the selection matrix $\mathrm{J}$ is then written:

$$
J=\left(\begin{array}{cc}
-1 & 0 \\
1 & 0
\end{array}\right)
$$

The model of compass is:

$$
\dot{q}=\left(\begin{array}{l}
\dot{\theta}_{n s} \\
\dot{\theta}_{s} \\
\ddot{\theta}_{n s} \\
\ddot{\theta}_{s}
\end{array}\right)=A\left(\begin{array}{l}
\theta_{n s} \\
\theta_{s} \\
\dot{\theta}_{n s} \\
\dot{\theta}_{s}
\end{array}\right)+B_{h}\left(\begin{array}{l}
T_{h} \\
T_{c}
\end{array}\right) \text { with } B_{h}=\left(\begin{array}{cc}
0 & 0 \\
0 & 0 \\
-1 & 0 \\
1 & 0
\end{array}\right)
$$


- The ankle is commanded: the selection matrix is:

$$
J=\left(\begin{array}{ll}
0 & 0 \\
0 & 1
\end{array}\right)
$$

The model command matrix B becomes:

$$
B_{c}=\left(\begin{array}{ll}
0 & 0 \\
0 & 0 \\
0 & 0 \\
0 & 1
\end{array}\right)
$$

- The ankle and the hip are commanded.

The selection matrix in this case becomes:

$$
J=\left(\begin{array}{cc}
-1 & 0 \\
1 & 1
\end{array}\right) \text { and } B_{c h}=\left(\begin{array}{cc}
0 & 0 \\
0 & 0 \\
-1 & 0 \\
1 & 1
\end{array}\right)
$$

In the case of the compass gait robot model, the application of the switching system approach has to find the set of feedback gain $K_{i}$, such as the closed loop system for a well determined delay is stable, has to take into account the command swaying between the hip, the ankle, or both at the same time.

\section{SimULATION RESUlTS}

The compass robot described the owing discrete time system, with:

$a=b=0.5 m, l=1 m, m=5 K g, m_{h}=10 K g, g=9.8 m / s^{2}$,

$\varphi=\alpha=3^{\circ}$

$\tau_{\min }=T_{e}, \tau_{\max }=6 T_{e}$

$\left\{\begin{array}{l}x(+1)=A_{d} x(k)+B_{d} u(k-\tau(k)) \\ y(k)=C_{d} x(k)\end{array}\right.$

Where:

$A_{d}=\left(\begin{array}{cccc}1 & -1.4 \mathrm{e}^{-07} & 0.0001 & -4.667 \mathrm{e}^{-012} \\ -2 \mathrm{e}^{-08} & 1 & -6.667 \mathrm{e}^{-013} & 0.0001 \\ -0.0026 & -0.0028 & 1 & -1.4 \mathrm{e}^{-07} \\ -0.0004 & -0.0014 & -2 \mathrm{e}^{-08} & 1\end{array}\right)$

Thematrix of control $B_{d}$ is depending in which part is controlled hip, ankle or hip and ankle.

$$
\begin{aligned}
& B_{d h}=\left(\begin{array}{cc}
-5 \mathrm{e}^{-009} & 0 \\
5 \mathrm{e}^{-009} & 0 \\
-0.0001 & 0 \\
0.0001 & 0
\end{array}\right), B_{d c}=\left(\begin{array}{cc}
0 & -1.167 \mathrm{e}^{-016} \\
0 & 5 \mathrm{e}^{-009} \\
0 & -4.667 \mathrm{e}^{-012} \\
0 & 0.0001
\end{array}\right), \\
& B_{d c h}=\left(\begin{array}{cc}
-5 \mathrm{e}^{-009} & -1.167 \mathrm{e}^{-016} \\
5 \mathrm{e}^{-009} & 5 \mathrm{e}^{-009} \\
-0.0001 & -4.667 \mathrm{e}^{-012} \\
0.0001 & 0.0001
\end{array}\right)
\end{aligned}
$$

$C_{d}=\left(\begin{array}{cccc}1 & 0 & 0 & 0 \\ 0 & 1 & 0 & 0 \\ 0 & 0 & 1 & 0 \\ 0 & 0 & 0 & 0\end{array}\right), D_{d}=0$

The commutation due to the control choice is known at real time. In other part the commutation law due to the time delay is considered as unknown in real time but bounded. So the difficulty is to determinate three different state feedback gains stabilizing the walk despite the delay and the switch between control positions.

The studies of the stability of the delay compass gait biped robot controlled with feedback state rely on the use of parameter dependent Lyapunov function. The formulation of the gain calculation problem has led to nonlinear matrix inequality. Allaying the relaxation method presented in partII lead to a LMI's conditions. The use of Matlab (c) for the resolution of LMI allows calculating three feedback gains $K_{d}$ (Table 1) ensuring the stability of the walking with an arbitrary delay affecting the input from one to six times the sampling period.

TABLE I. GAINS $K_{d}$

\begin{tabular}{|l|l|}
\hline Hip Command: $\boldsymbol{B}_{d}=\boldsymbol{B}_{d h}$ \\
\hline$K_{d H}=1.0 \mathrm{e}+08 *\left[\begin{array}{cccc|}0.9516 & -1.2486 & 0.5235 & -0.2266 \\
0 & 0 & 0 & 0\end{array}\right]$ \\
\hline Ankle Command: $\boldsymbol{B}_{d}=\boldsymbol{B}_{d c}$ \\
\hline$K_{d C}=1.0 \mathrm{e}+09 *\left[\begin{array}{cccc}0 & 0 & 0 & 0 \\
0.4100 & -1.5177 & -0.4661 & 1.5738\end{array}\right]$ \\
\hline Hip and Ankle) Command: $\boldsymbol{B}_{d}=\boldsymbol{B}_{d c h}$ \\
\hline$K_{d C H}=1.0 \mathrm{e}+07 *\left[\begin{array}{cccc}1.2906 & -0.9221 & -0.9084 & 0.5399 \\
-1.3848 & 1.1161 & 0.6100 & -0.3413\end{array}\right]$ \\
\hline
\end{tabular}

The simulation of the system around the balance point for various command matrices $K_{d}$, applied at the level of the ankle and of the hip, gives the following signals:(fig2, fig3, fig4)

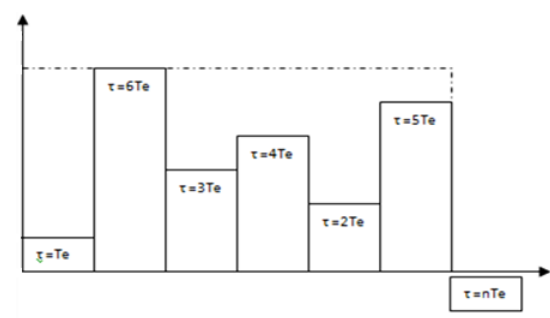

Fig. 3. Switching of the command according to the delays

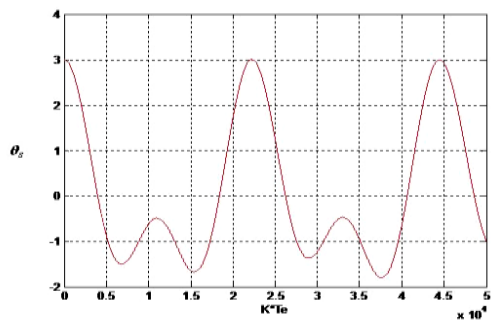

Fig. 4. $\theta_{s}$ : Angle of the leg when it touch the ground 


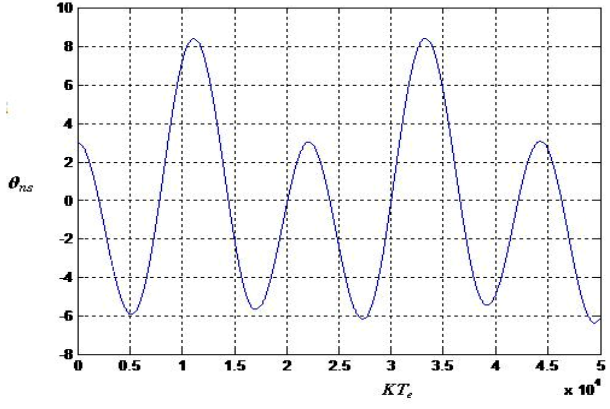

Fig. 5. $\theta_{n s}$ : Angle of the leg during flight

According to the curves of signals, $\theta_{s}, \theta_{n s}$, the system remains stable, the gains by state feedback $K_{i}$ calculated by the switching system method stabilizing the closed loop system for a delay reaching six times the sampling period in the three cases of command swaying. This calculus method offers thus an important stability domain because it tolerates the uncertainties on the system model aspect in real time. The simulation under Matlab simulink of the compass model verifies the compass stability conditions before the flight: $\theta_{n s}+\theta_{s}=-2 \varphi$ and $\theta_{n s}+\theta_{s}=2 \alpha$, and the condition after one step movement: $\theta_{n s}{ }^{-}-\theta_{s}{ }^{-}=\theta_{s}{ }^{+}-\theta_{n s}{ }^{+}=2 \alpha$. for the various gains of $K_{d}$ command.

\section{CONCLUSION}

We are interested in this product to the application of switched system approach to the compass robot control, controlled via a data transmission network seat of delays which prevent the establishment of control sequences in real time. Specifically, we studied the stability of the switched system of this model, when the transmitted control switches between the hip, ankle, hip or ankle at the same time. The simulation results justified the stability of the robot model for a delay varying from one to six times sampling period.

\section{REFERENCES}

[1] Yildirim Hurmuzlua, Frank Genotb, Bernard Brogliatoc, Modeling, stability and control of biped robots - a general frame work.Automatica 40 (2004) 1647 - 1664.
[2] A. Chemori and A. Loria, commande d'un robot bipède sur un cycle complet de marche. CIFA (Conférence international francophone d'automatique). France, 2002.

[3] Arouri, W., Maherzi, E., Besbes, M., Safya Belgith. Switched Control for the walking of a Compass Gait Biped Robot, Research Journal of Applied Sciences, Engineering and Technology. 2014.

[4] L. Hetel. Stabilité et commande robuste des systèmes à commutation, Institut National Polytechnique de Lorraine.2007.

[5] Seuret, Alexandre. Commande et observation des systèmes à retards variables : théorie et application. Thèse de doctorat. Universités des Sciences et technologie de Lille, école centrale de Lille, 2006.

[6] Fridman, E. and U. Shaked, 2002. An improvedstabilization method for linear time-delay systems, IEEE Trans. Automatic Control, 47: 19311937.DOI: 10.1109/TAC.2002.804462

[7] Cloosterman, M.B.G., N.V.D. Wouw, W.P.M. Heemelsand H. Nijmeijer, 2007. Stability of networkedcontrol systems with large delays. Proceedings ofthe 46th IEEE Conference on Decision andControl, Dec. 12-14, IEEE Xplore Press, NewOrleans, LA., pp: 5017-5022. DOI:10.1109/CDC.2007.4434669

[8] Ariba, Y. and F. Gouaisbaut, 2007. Delay-dependentstability analysis of linear systems with timevaryingdelay. Proceedings of the 45th IEEEConference on Decision and Control, Dec. 12-14,IEEE Xplore Press, New Orleans, LA., pp: 2053-2058. DOI: 10.1109/CDC.2007.4434619

[9] L.Hetel, J. Daafouz, and C. Iung. Stability analysis for discrete time switched systems with temporary uncertain switching signal. In Proceedings of 46th IEEE Conference on Decision and Control, 2007.

[10] Daafouz J., Riedinger P.et lung C. Stability analysis and control synthesis for switched systems: A switched lyapunov function approach.IEEE transactions on automatic control vol $47 \mathrm{n}^{\circ} 11,2002, \mathrm{p}$ 1883-1887.

[11] Daafouz, J., G. Millerioux and C. Lung, 2002b. A polyquadraticstability based approach for linearswitched systems. Int. J. Control, 75: 13021310.DOI: 10.1080/0020717021000023735

[12] Daafouz, J. and J. Bernussou, 2001. Parameterdependent Lyapunov functions for discrete timesystems with time varying parametricuncertainties. Syst. Control Lett., 43: 355-359. DOI: 10.1016/S0167-6911(01)00118-9.

[13] L. Hetel, J. Daafouz, and C.Iung. Equivalence between the lyapunovKrasovskii functional approach for discrete delay system and the stability conditions for switched system. Nonlinear Analysis Hybrid Systems 2(3):697-705.

[14] Maherzi, E., J. Bernussou and R. Mhiri, 2007. Stabilityand stabilization of uncertain switched systems, apolyquadratic Lyapunov approach. Int. J. Sci.Techniques Automatic Control, $1:$ 61-74.

[15] Ahmed Keramane. Cycles de marche des robots de type compas, Analyse et commande. Thèse à l'Institut National Polytechnique de Greno. 